\title{
Inusual riqueza, composición y estructura arbórea en el bosque de tierra firme del Pongo Qoñec, Sur Oriente peruano
}

\section{An unusual richness, structure and composition tree in terra firme forest of Pongo Qoñec, Peruvian South East}

\author{
Isau Huamantupa-Chuquimaco
}

Herbario Vargas (CUZ), Facultad de Biología, Universidad Nacional San Antonio Abad del Cusco, San Antonio Abad del Cusco, Prolongación Av. de la Cultura s/n, Cusco, Perú. Email: andeanwayna@gmail.com

Presentado: $\quad 01 / 08 / 2009$ Aceptado: $\quad$ 23/08/2010 Publicado online: $14 / 12 / 2010$

\begin{abstract}
Resumen
Se estableció una parcela de 1 ha, en bosque de tierra firme en el Pongo de Qoñec, valle Kosñipata (Cusco), dentro de la Reserva Biósfera del Manú, a 710 m; $12^{\circ} 53^{\prime} 53^{\prime \prime S}$ y $72^{\circ} 22^{\prime} 25^{\prime \prime W}$. Se registró 56 familias, 153 géneros, 249 especies con 813 individuos con Dap $\geq 10 \mathrm{~cm}$. Las familias, más ricas fueron: Fabaceae (32 especies), Moraceae (24), Rubiaceae (17) y Lauraceae (12); los géneros más ricos, Inga (17), Neea (7), Miconia (5); se halló un área basal total de $40,50 \mathrm{~m}^{2}$, de ellas Iriartea deltoidea con $6,58 \mathrm{~m}^{2}$, Socratea salazarii 2,28 $\mathrm{m}^{2}$, y Endlicheria sp 1,65 $\mathrm{m}^{2}$, obtuvieron mayor ab; la especie con mayor valor de importancia fue: I. deltoidea, corroborando estudios anteriores en bosques de tierra firme. El análisis estructural muestra que clases diamétricas 10-20 cm, abarcan 71,3\% del total de fustes. Al comparar nuestros resultados, el Pongo de Qoñec, sorprendentemente supera en riqueza a parcelas adyacentes dentro del sur peruano y muestra un patrón de riqueza de familias y géneros similar a parcelas peruanas diversas como Yanamono 300 especies y Mishana (289), en Loreto. Consideramos que esta inusual riqueza se debe en gran medida a factores ambientales únicos que se dan en este Pongo, como son la alta precipitación, dinámica de vientos y la variabilidad de suelos en áreas pequeñas. Por tanto estos resultados difieren considerablemente de enunciados conocidos, que consideran
\end{abstract} a la amazonia sur peruana con moderada a baja riqueza arbórea.

Palabras Clave. Riqueza, diversidad, estructura, composición, Pongo de Qoñec.

Abstract

One plot of $1 \mathrm{ha}$, was established in the terra firm forest in the Pongo de Qoñec, Kosñipata valley (Cusco), inside the reserve Biósfera of the Manú, to $710 \mathrm{~m} ; 1^{\circ} 53^{\prime} 53^{\prime \prime S}$ and $72^{\circ} 22^{\prime} 25^{\prime \prime} \mathrm{W}$. We registered, 56 families, 153 genus, 249 species, with 813 individuals with Dap $\geq 10 \mathrm{~cm}$. Families, more rich were: Fabaceae (32 species), Moraceae (24), Rubiaceae (17) and Lauraceae (12); The most rich genus were, Inga (17), Neea (7), Miconia (5); the total basal area was $40.50 \mathrm{~m}^{2}$, of them Iriartea deltoidea with $6.58 \mathrm{~m}^{2}$, Socratea salazarii $2.28 \mathrm{~m}^{2}$, and Endlicheria sp $1.65 \mathrm{~m}^{2}$, obtained bigger ab; The specie with most high value of importance went: I deltoidea, corroborating previous studies in the terra firm forests. The structural analysis evidences than diametrics class of $10-20 \mathrm{~cm}$, was $71.3 \%$ of the total of individuals. When comparing our results, the Pongo Qoñec, surprisingly obtained high richness to adjacent plots, inside the Peruvian south and sample a similar patterns of richness of families and genus to most diversity Peruvian plots how Yanamono 300 species and Mishana (289), in Loreto. We considered that these unusual richness are due to environmental factors, present in the Pongo de Qoñec, such as the high precipitation, dynamics of winds and the variability of soils in little areas. Therefore these results differ considerably from known enunciates, that consider that Amazon south Peruvian have low and moderate trees richness.

Keywords. Richness, diversity, structure, composition, Pongo de Qoñec.

\section{Introducción}

Las más altas cifras de riqueza y diversidad del mundo son registradas para la Amazonía neotropical, fundamentalmente en la parte oeste; como el caso de parcelas cercanas a Iquitos (Perú), con diversidades entre 280 a 300 especies, con Dap mayores a $10 \mathrm{~cm} /$ ha, o aquellas en Yasuní (Ecuador) con 253 a 283 especies (Gentry 1988a, 1988b, Phillips et al. 1994, Ter Steege at al. 2000, Pitman 2000, Salgado et al. 2004, Valencia et al. 2004). La zona sur de la Amazonia del Perú (Madre de Dios, Cusco), es conocida por presentar una riqueza y diversidad de árboles leñosos entre moderada a baja (Gentry 1988a, 1988b, Phillips et al. 1994).

Por otro lado existen pocos estudios con parcelas permanentes en bosques de tierra firme en rangos altitudinales por encima de los $400 \mathrm{~m}$. El área estudiada en el presente trabajo se encuentra dentro de la zona cultural perteneciente a la reserva de Biosfera del Manú, donde se halla el Pongo de Qoñec; una formación geológica donde en una corta gradiente altitudinal se aprecian cambios de composición edáfica, con suelos de diferente composición de nutrientes y textura (INRENA \& PRO-MANU 2004). El presente trabajo da a conocer, la composición y estructura arbórea, y compara la zona de estudio con aéreas adyacentes y distantes.

\section{Material y métodos}

\section{Área de estudio}

La zona de estudio está ubicada en el sector Qoñec en la margen derecha del río Alto Madre de Dios a $7 \mathrm{~km}$ de la localidad de Pillcopata; entre los 700 a $720 \mathrm{~m}$ de altitud (12 $53^{\prime} 53^{\prime \prime}$, $72^{\circ} 22^{\prime 2} 5^{\prime \prime W)}$; y pertenece a la Zona de Transición Amazónica de la Reserva de la Biósfera del Manú, dentro del Distrito de Kosńipata, Provincia Paucartambo y Departamento del Cusco.

La zona de estudio corresponde al bosque muy húmedo premontano Sub tropical. La temperatura presenta una media anual de $23,97^{\circ} \mathrm{C}$ y las precipitaciones un promedio anual de $3914,9 \mathrm{~mm}$. Con una marcada estacionalidad, con meses de lluvias torrenciales entre diciembre y abril (INRENA \& PROMANU 2004).

La fisiografía está conformada por las últimas estribaciones de la cadena montañosa del Paucartambo, llegando hasta el arco del Fiztcarral y parte de la cadena del Pantiacolla, el cual comprende el Pongo de Qońec. Los suelos en la zona de estudio son clasificados en la región geoedafológica acrisólica, de origen entre el paleozoico superior y el terciario superior (aproximadamente 250 - 15 millones de años) (INRENA \& PRO-MANU 2004). 


\section{Instalación de la parcela permanente}

Se instaló y evaluó una parcela permanente de 1 ha, siguiendo los protocolos de RAINFOR (Phillips \& Baker 2002), y el análisis de datos basados en variables de estructura y composición (Matteuci \& Colma 1982), ambas se describen a continuación.

La ubicación se hizo considerando características topográficas, la homogeneidad del bosque, tipo de suelo y acceso adecuado. La parcela de una hectárea, fue de $40 \mathrm{~m}$ ancho y $250 \mathrm{~m}$ largo, dividido en subparcelas de 20 × $20 \mathrm{~m}\left(400 \mathrm{~m}^{2}\right)$. Se colocaron estacas plásticas en los extremos de las subaparcelas, y se delimitaron con una pequeña trocha al borde de la parcela. Los individuos evaluados fueron árboles y lianas a 1,30 m de POM (Punto óptimo de medida). Finalizada el plaqueo y toma de datos de los cuadrantes se procedió a realizar las colecciones para su posterior identificación y depósito en el herbario (CUZ) de la Universidad Nacional de San Antonio Abad del Cusco y el (MO) del Missouri Botanical Garden, USA.

\section{Análisis de datos}

Para el análisis de los datos se hallaron parámetros estadístico poblacionales de dominancia, densidad y frecuencia, de estas se obtuvieron los valores relativos y absolutos, con las que se procedió hallar el índice de valor de Importancia (IVI) para las especies y familias; también se calculo el área basal de la parcela y las diferentes taxas. (Matteuci \& Colma, 1982).

La clasificación taxonómica tanto de familias y géneros, se baso en la propuesta por el APG II (2003).

\section{Resultados y discusión}

\section{Riqueza y abundancia florística}

En la parcela de una hectárea del bosque de tierra firme Pongo de Qońec se registró 56 familias con 153 géneros, 249 especies, con un total de 813 individuos, correspondientes a 810 árboles y 3 Lianas con Dap $\geq 10 \mathrm{~cm}$. Dentro de ellas las 11 familias más ricas y diversas fueron: Fabaceae (32 especies), Moraceae (24), Rubiaceae (17), Malvaceae (13), Lauraceae (12), Euphorbiaceae (11), Urticaceae (9), Flacourtiaceae, Annonaceae, Myristicaceae y Nyctaginaceae con (7 especies), agrupando el 58\% del total.

Al igual que estudios similares de selva baja, el Pongo de Qoñec muestra a las Fabaceae, Moraceae y Rubiaceae como las más ricas en especies, coincidiendo con la mayoría de parcelas, por ejemplo Pitman et al. (2002), menciona para 13,8 ha, de parcelas en Manú, a Fabaceae, Moraceae, Annonaceae y Euphorbiaceae como las más ricas. También en el norte peruano (Allpahuayo y Yanamono); Gentry (1988a, 1988b); Phillips et al. (1994) y Vásquez y Phillips (2000); al analizar parcelas ubicadas dentro de los llamados varillales en zonas con arena blanca, con altos endemismos locales, reportan a Fabaceae, Moraceae, Lauraceae y Annonaceae, como las más ricas.

Otra zona también en el norte del Perú, Jenaro herrera-Loreto, en un bosque de terraza alta pero con suelo más arcilloso, muestra a Lecythidaceae, Sapotaceae, Fabaceae y Lauraceae como las más ricas (Honorio et al. 2008), contrastando con lo observado en nuestra parcela Lecythidaceae y Sapotaceae tienen pocas especies, (2 y 3). En general todas estas familias son consideradas comunes y dominantes en bosques de tierra firme, principalmente para la Amazonía del oeste, tanto en la zona sur y centro de Sudamérica (Ter Steege at al. 2000, Pitman et al. 2002).
En el pongo de Qońec la alta riqueza observada en las familias Rubiaceae y Lauraceae podría deberse en gran medida a la mezcla o traslape de poblaciones de Amazonía baja y bosques montañosos, considerando que estas cifras son mayores a parcelas cercanas, como las de Pakitza y Cocha Cashu (Pitman et al. 1999).

La parcela de San Pedro (Palomino 1997) cercana al área de estudio pero a $1700 \mathrm{~m}$, presenta similitud en la composición de taxas considerando a Moraceae como la más rica con 21 especies seguida de Euphorbiaceae (20), Melastomataceae y Lauraceae.

Las familias más abundantes fueron: Fabaceae con (102 individuos), Urticaceae (87), Arecaceae (79), Euphorbiaceae (59), Moraceae (42), Polygonaceae (35), Nyctaginaceae (32), Rubiaceae (30), Malvaceae (27), Flacourtiaceae (26); estas diez familias agrupan el 63,9\% del total de individuos, siendo el promedio de 14 individuos para cada una.

Se halló 19 familias con una sola especie que representa el $32 \%$ del total, este porcentaje supera en un $10-15 \%$ a parcelas amazónicas y de montańas, y esta incrementada por la presencia de especies poco frecuentes, como de las familias: Thymelaeaceae, Lepidobothryaceae, Vochysiaceae, Theophrastaceae y Solanaceae; pero algunas de estas especies presentan numerosos individuos como es el caso de Vochysia kosnipatae, especie bastante común en este valle y ampliamente utilizada como maderable.

Los géneros más ricos fueron, Inga (17 especies), Neea (7), Miconia, Guarea, Sterculia y Virola (5); Brosimum, Casearia, Cecropia, Naucleopsis, Nectandra, Protium y Sloanea con (4), estos 13 géneros suman 76 especies y agrupan el 29\% del total de especies; muestran similitud a los registrados en Cocha Cashu, donde Inga abarca cerca de 30 especies; de igual manera este género es frecuentemente la más diversa en parcelas de zonas bajas y de bosques más montañosos. Miconia en este caso es diferente a lo que comúnmente se registra en amazonía, tal vez debido a que en el pongo de Qońec descienden especies de boques más altos como el de San Pedro, donde es el segundo género más rico con 6 especies y en San Alberto aún más alta $(2200 \mathrm{~m})$, con 9 especies (Gómez 2000). Neea con 7 especies, se muestra superior a otras parcelas, Guarea, Sterculia y Virola, muestran especies frecuentes en parcelas adyacentes y lejanas como en Loreto.

Con Jenaro Herrera se diferencia ampliamente, por el género Eschweilera donde es más abundante (Honorio et al. 2008), por estar condicionada a la presencia de suelos pobres en nutrientes, contrariamente en nuestra zona de estudio, solo Eschweilera coriacea está presente con un individuo.

Pitman et al. (2008), en un estudio a lo largo de zonas bajas de los Andes (Ecuador) hasta áreas cercanas al rio Amazonas (Iquitos - Perú y Manaus-Brasil), muestra a cinco géneros comunes: Eschweilera (5,2\% del total de individuos), Inga, Virola, Iriartea y Protium, los que demuestran a excepción de Eschweilera, la amplia amplia distribución de estos en la Amazonía neotropical.

Otro género ampliamente común en parcelas de zonas bajas es Virola e Iryanthera (Myristicaceae), de las cuales Virola en Qoñec es una de las más ricas con 5 especies, e Iryanthera no presenta registro alguno.

Como en casi todas las parcelas de tierra firme en la Amazonía sudamericana, fundamentalmente en la base de los Andes, en nuestro estudio la palmera Iriartea deltoidea fue la más abundante y dominante con 41 individuos (5,04\%), y con el más alto valor 
de importancia $(33,92)$, corroborando los estudios de Pitman et al. $(2001,2002,2008)$, entre otros. Pero en parcelas también del norte peruano pero lejanas a las estribaciones de los andes como: Mishana-Allpahuayo y Jenaro Herrera la palmera dominante fue Oenocarpus batahua, especie preferente de suelos aparentemente pobres y temporalmente inundables.

Otra especie abundante lo constituye Socratea salazarii con 28 individuos, que es poco frecuente en parcelas, ya que aparentemente prefiere suelos más ricos y bosques montañosos.

La presencia de especies de rápido crecimiento como: $P$, tomentosa, T. peruviana, Tachigali aff. polyphylla, Croton sampatik (19 ind); se debe probablemente al considerable dinamismo que muestra el pongo de Qoñec por la presencia de altas precipitaciones $(3900 \mathrm{~mm})$, acompańadas de fuertes vientos huracanados.

Otras ampliamente abundantes en el Manú como: Rinorea guianensis, Astrocaryum murumuru, Pseudolmedia laevis, Pausandra trianae, Leonia glycycarpa, y Celtis schippii, en el pongo de Qoñec también están presentes pero con pocos individuos y menor densidad.

Otra especie importante presente en Qoñec proveniente de bosques montanos es el helecho arborescente Cyathea caracasana con 9 individuos, que en parcelas de San Pedro y otras de montańas son comunes confirmando su adaptabilidad a zonas más bajas.

Se encontró un total de 108 especies con un solo individuo, lo que representa el $41,7 \%$ del total de especies evaluadas. Comparando con otras localidades como Jenaro Herrera, el $50 \%$ son especies con un solo individuo; para San Alberto se reporta $40,38 \%$ de especies con un solo individuo, cifra similar al Pongo de Qońe y para la localidad de Yanamono se tiene 63\% especies con un solo individuo, lo que supera notablemente al Pongo de Qoñec en 38 especies.

El número de especies presentes en Qoñec es semejante al reportado en otras parcelas de alta riqueza en el Perú (Tabla 1), como Yanamono con 300 especies y Mishana con 289 (Gentry 1988a, 1988b), ambas en Loreto. Sin embargo presenta mayor riqueza a parcelas del norte peruano como Jenaro Herrera, que en parcelas de terraza alta reportan como máximo 193 especies (Honorio et al. 2008), similar cifra mantienen Cocha Cashu y otras parcelas en el Manú con 126 y 200 especies (Pitman et al. 1999, 2001), o en el bosque montano de San Pedro (1700 $\mathrm{m})$, con 192 especies.

Tabla 1. Comparación de la riqueza de taxones con parcelas de Tierra firme en la Amazonía Peruana y zonas adyacentes.

\begin{tabular}{lcccc}
\hline Localidad & $\begin{array}{c}\text { Altitud } \\
(\mathrm{m})\end{array}$ & Familias & Géneros & Especies \\
\hline Pongo Qoñec** $^{*}$ & 710 & 56 & 153 & 249 \\
Yanamono* $^{*}$ & 140 & & & 300 \\
Allpahuayo* $^{*}$ & 150 & & & 289 \\
San Pedro** & 1700 & 45 & 91 & 192 \\
Manú $^{* *}$ & $250-400$ & 43 & & $126-200$ \\
Chalalan*** $^{*}$ & 400 & 43 & & 118 \\
\hline
\end{tabular}

Parcelas del Norte Peruano *(Gentry 1988a, 1988b), Parcelas del Sur Peruano **(Pte. Estudio; Palomino 1997; Pitman et al. 1999, 2001), Parcela del Norte Boliviano ***(Silman et al. 2005).
Tabla 2. Comparación de densidad y áreas basales, con parcelas de Tierra firme en la Amazonía Peruana y zonas adyacentes

\begin{tabular}{lccc}
\hline Localidad & Fustes & Lianas & Área basal total $\left(\mathbf{m}^{2}\right)$ \\
\hline Pongo Qoñec** & 813 & 3 & 40,50 \\
Yanamono* $^{*}$ & 580 & 26 & 32,7 \\
Allpahuayo* $^{*}$ & $608-616$ & $27-26$ & $27-26$ \\
Mishana * $^{*}$ & 842 & 16 & 28,7 \\
San Pedro** & 618 & 1 & 23 \\
Manú $^{* *}$ & 598 & $17-45$ & $25-29$ \\
Chalalan*** & 702 & & 38,8 \\
\hline
\end{tabular}

Parcelas del Norte Peruano *(Gentry 1988a, 1988b), Parcelas del Sur Peruano **(Pte. Estudio; Palomino 1997; Pitman et al. 1999, 2001), Parcela del Norte Boliviano ***(Silman et al. 2005).

Sin duda esta alta riqueza de especies, géneros y familias se debe a la confluencia observada de los dos tipos de bosque mencionados y a la mezcla aparente de suelos ricos y pobres en nutrientes, observándose afloramientos de roca madre y rocas (aparentemente compuestas de diatomeas), quebradas fácilmente. Por tanto estos patrones de riqueza atípicos difieren ampliamente los conocidos enunciados de autores como Gentry (1988a, 1988b); Phillips (1994), Pitman et al. (1999, 2001), quienes enfatizan que la riqueza de árboles es alta en bosques adyacentes a la línea ecuatorial y existe disminución marcada de la diversidad a medida que aumenta la gradiente latitudinal al sur.

\section{Análisis estructural}

Número total de individuos.- La cifra de 813 individuos con Dap $\geq 10 \mathrm{~cm}$ (Tabla 2), registrada en la parcela constituye una de las más altas en toda la Amazonía peruana, mostrando patrón similar a la parcela de Mishana - Iquitos (Gentry 1988a, 1988b), con 842 individuos. Parcelas de Madre de Dios y adyacentes engloban entre 550 - 673 individuos en promedio, que también se aprecia en otras parcelas del Norte - Yanamono y Jenaro Herrera. Estas cifras están directamente asociadas a la dinámica del ecosistema y naturaleza de los suelos, es así que en los llamados Varillales (Gentry 1988a, 1988b, Phillips et al. 1994, Vasquez \& Phillips 2000), registran altos porcentajes de fustes entre los $10-20 \mathrm{~cm}$ de Dap, los cuales evidencian preferencia por suelos pobres de arena blanca.

La presencia de solamente tres individuos de lianas constituye una característica poco frecuente dado que normalmente se catalogan para zonas bajas mayores a 15 individuos, las cuales son habituales hasta los $1000 \mathrm{~m}$, y relativamente ricos principalmente en las Bignoniaceae. (Gentry 1988, 1988b).

Área basal.- El área basal total para la parcela fue de 40,50 $\mathrm{m}^{2}$. Con las familias, Arecaceae $\left(7,36 \mathrm{~m}^{2}\right)$, Urticaceae $\left(4,56 \mathrm{~m}^{2}\right)$, Fabaceae $\left(2,18 \mathrm{~m}^{2}\right)$, Euphorbiaceae $\left(1,18 \mathrm{~m}^{2}\right)$, Lauraceae $(1,30$ $\left.\mathrm{m}^{2}\right)$, de mayor área basal y las especies más representativas: $I$. deltoidea $\left(6,58 \mathrm{~m}^{2}\right)$, S. salazarii $\left(2,74 \mathrm{~m}^{2}\right)$, Endichleria $p$ (1,65 $\left.\mathrm{m}^{2}\right)$, T. aff polyphylla $\left(1,20 \mathrm{~m}^{2}\right)$, P. tomentosa $\left(1,18 \mathrm{~m}^{2}\right)$, P. cecropiifolia $\left(1,15 \mathrm{~m}^{2}\right)$, T. peruviana $\left(0,91 \mathrm{~m}^{2}\right)$, Porcelia nitidifolia $\left(0,86 \mathrm{~m}^{2}\right)$, $N$. divaricata $\left(0,79 \mathrm{~m}^{2}\right), C$. sampatik $\left(0,75 \mathrm{~m}^{2}\right)$, constituyendo el $66,8 \%$ del total de individuos, las que hacen un total $14,96 \mathrm{~m}^{2}$, representando el $36,9 \%$ del total de la parcela. El área basal total de $40,50 \mathrm{~m}^{2}$, difiere a los obtenidos en la mayoría de tierras bajas que van desde los $24-32,7 \mathrm{~m}^{2}$. Chalalan en Bolivia, muestra una alta cifra de $38,8 \mathrm{~m}^{2}$ de área basal, con alto porcentaje de individuos de $I$. deltoidea con 265 fustes (Silman et al. 2005). 


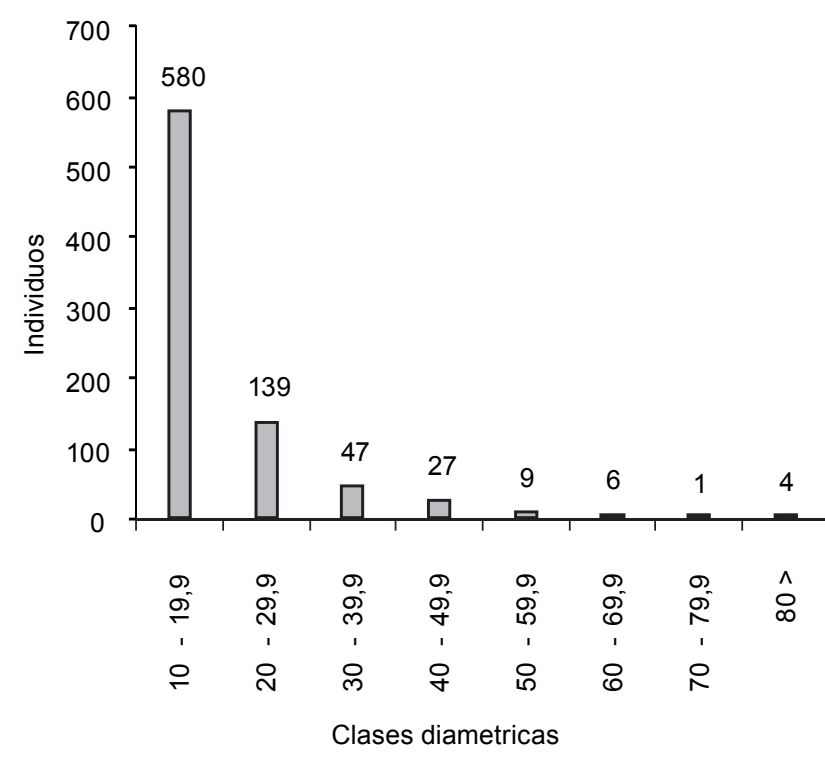

Figura 1. Distribución de individuos según clases diamétricas.

Distribución de diámetros.- Se registró 580 fustes (Fig. 1), que corresponden a clases diamétricas de 10 - 19,9; agrupando al 71,3\% del total, patrón frecuente en todas las parcelas, para nuestra parcela es una tanto mayor por la presencia de especies de palmeras y helechos en gran número, siendo $S$. salazarii, $S$. exorrhiza, Wettinia weberbaueri y $C$. caracasana. Clases de 60 - 89,9 engloban a 11 individuos, constituyendo el 1,35\% del total, donde el diámetro mayor es de 84,5 (Inga sp1); al compararse se muestra el mismo patrón de distribución con otras parcelas, por ejemplo en Cocha Cashu constituyen el 63,15\% de individuos; Yanamono el 60,99\% de individuos; Mishana el 69,7\% de individuos (Gentry 1988a, 1988b), similar a nuestra área de estudio; para la zona de bosque montano, San Alberto $73,94 \%$ y para San Pedro se tiene 388 individuos equivalente al $62,76 \%$.

Distribución de alturas.- Dentro de la parcela las alturas obtenidas oscilan desde un rango de 3-40 m (Fig. 2), donde el rango con mayor número de individuos fue de $11-20 \mathrm{~m} \mathrm{(381}$

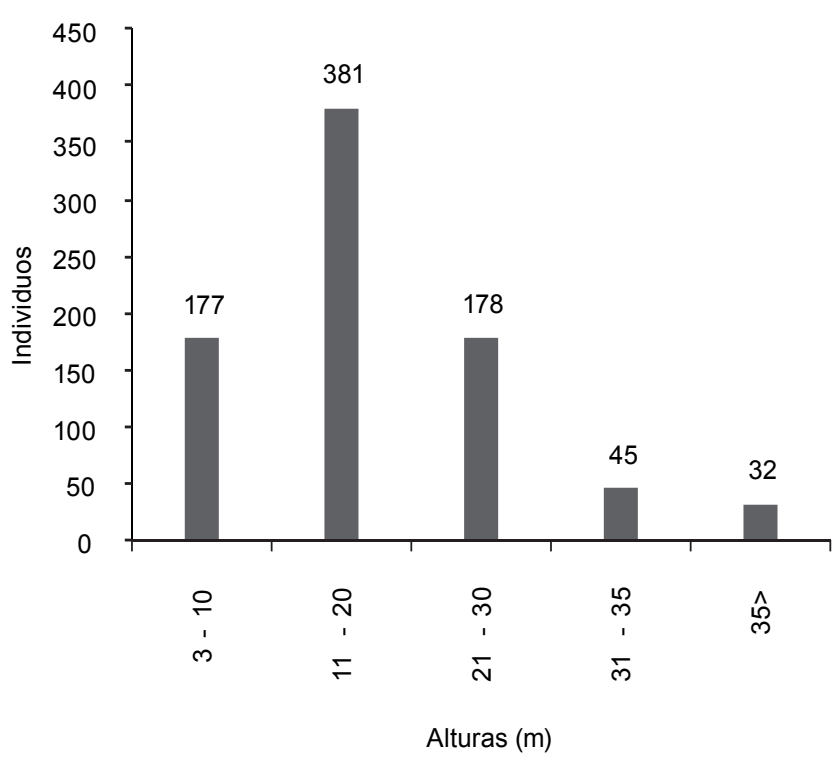

Figura 2. Distribución del número de individuos según clases de alturas. individuos, 28,9\%), el rango de altura con menor número de individuos fue de $>35 \mathrm{~m}$ (32 individuos, 1,72\%); Tabebuia serratifolia y Chimarrhis hookerii fueron las especies de mayor altura. Cyathea caracasana, Alsophylla sp. y Dicksonia sellowiana fueron las de menor altura (1,84\% del total); estas especie en bosques montanos llegan a medir más de $12 \mathrm{~m}$, como en San Pedro y San Alberto. Estas alturas de los árboles difieren en menor proporción con otras parcelas de selva baja donde algunas especies llegan a medir hasta $65 \mathrm{~m}$, (bosques maduros), teniendo una muestra con reportes de especies con mayor altura y diámetro en parcelas de Cocha Cashu (Pitman et al. 1999, Pitman 2001).

Conclusiones.- Consideramos que esta alta riqueza de taxas halladas en el Pongo de Qoñec, sin duda pone en discusión conceptos bastante conocidos y aceptados por varios autores como Gentry (1988a, 1988b), Phillips (1994)y Pitman et al. $(1999,2001)$ que afirman la existencia de una baja a moderada riqueza de familias, géneros y especies para el sur de la Amazonía peruana, ya que entre otros conceptos enfatizan que la riqueza de árboles es alta en bosques adyacentes a la línea ecuatorial y existe disminución marcada de la diversidad a medida que aumenta la gradiente latitudinal al sur.

A la vez, creemos que estos patrones atípicos están relacionados con la variación amplia de composición de suelos en áreas pequeñas por la mezcla de suelos ricos y pobres en nutrientes, el rápido cambio de microclimas, gradientes topográficos y altas concentraciones de precipitación observado en el valle de Kosñipata. Otro factor importante que creemos son la fluctuación de los vientos provenientes de zonas más bajas jugando un papel importante en la dinámica del bosque, tal como lo sugieren Phillips et al. (1994); Ter Steege et al. (2000) y recientemente Killeen et al. (2007). Por otra parte desconocemos otros procesos biológicos asociados a la riqueza y diversidad, como los efectos provocados por los depredadores, dispersores, la competencia entre plantas (Givnish 1999), que podrían ayudar a comprender muchos conceptos.

\section{Agradecimientos}

La presente publicación forma parte de la tesis realizada para optar al título de Biólogo en la Universidad Nacional San Antonio Abad del Cusco. Por lo cual Agradecemos al Ing. Rodolfo Vásquez, por habernos facilitado desinteresadamente equipos de campo de herbario e impresión final. Al Msc. Abel Monteagudo, por su apoyo en la identificación de algunos grupos taxonómicos. A mis padres Isaac y Claudia, hermanos Judith, Abner y Lincol por haberme apoyado en el muestreo de campo y herbario, a mis amigos y compañeros Elías Paz, Eduar Berríos y Heber Huamantupa por su ayuda en el trabajo de campo. A los señores Juvenal Gutiérrez, Hugo Sánchez y Florentino Huamantupa por la información de los usos y nomenclatura vernácular de las especies en estudio.

\section{Literatura citada}

APG II. 2003. An update of the Angiosperm Phylogeny Group, Classification for the orders and families of Flowering plants: Botanical Journal of the Linnean Society 141: 399-436.

Gentry A.H. 1988a. Changes in plant community diversity and floristic composition on environmental and geographical gradients. Ann. Mo. Bot. Gard. 75: 1-34.

Gentry A.H. 1988b. Tree species richness of upper Amazonian forests. Proc. Natl. Acad. Sci. USA 85: 156-159. 
Gentry A.H. \& R. Ortiz. 1993. Patrones de composición florística en la amazonia peruana. 155-166 in R. Kalliola, M. Puhakka, and W. Danjoy, editors. Amazonia Peruana: vegetación húmeda tropical en el llano subandino. Paut \& Onern, Jyvaskyla, Finland.

Givnish T.J. 1999. On the causes of gradiens in tropical tree diversity. Journal of Ecology 87: 193 - 210.

Gómez D. 2000. Composición Florística en el Bosque ribereño de la Cuenca Alta San Alberto, Oxapampa - Perú; Tesis para optar al título de Ingeniero Forestal. Universidad Nacional Agraria la Molina. Lima - Perú. 230pp.

Honorio E.N., T.R. Pennignton, L.A. Freitas, et al. 2008. Análisis de la Composición florística de los bosques de Jenaro Herrera, Loreto, Perú. Rev. Peru. Biol. 15(1): 53 - 60.

Killen T.J., M. Douglas, T. Consiglio, P.M. Jørgensen \& J. Mejia. 2007. Dry spots and wet spots in the Andean hotspot. Journal of Biogeography 34: 1357-1373.

Leigh E.G., P. Davidar, C.W. Dick, et al. 2004. Why Do Some Tropical Forests Have So Many Species of Trees?. BIOTROPICA 36(4): 447 - 473

Matteuci D. \& A. Colma. 1982. Metodologías para la evaluación de la vegetación. Monografía de la secretaria general de la OEA. Washington. D.C. USA.

Palomino P.W. 1997. Diversidad y Asociación arbórea en el bosque nublado de San Pedro, Reserva de Biosfera del Manú. Tesis para optar al titulo de Biólogo. Universidad Nacional de San Antonio Abad del Cusco.

Phillips O.L., P. Hall, A.H. Gentry, et al. 1994. Dynamics and species richness of tropical rain forests. Proc. Natl. Acad. Sci, USA. 91: $2805-2809$.

Phillips O. \& T. Baker. 2002. Field Manual for Plot Establishment and Remeasurement. Publicado en el Boletín de la Sociedad Geográfica de Lima 113: 2000.
Pitman N.C.A., J. Terborgh \& M.R. Silman. 1999. Tree species distributions in an upper Amazonian forest. Ecology 80:2651-2661.

Pitman N.C.A., J. Terborg, M.R. Silman, et al. 2001. Dominance and Distribution of tree species in upper Amazonian terra firme forests. Ecology 82: 2101-2117.

Pitman N.C.A., J. Terborgh, M.R. Silman, et al. 2002. A comparison of tree species diversity in two upper Amazonian forests. Ecology 83:3210-3224.

Pitman N.C.A., H. Mogollon, N. Dávila., et al. 2008. Tree Community Change across $700 \mathrm{~km}$ of Lowland Amazonian Forest from the Andean Foothills to Brazil. BIOTROPICA. 10.1111: 1744-7429

INRENA(Instituto Nacional de Recursos Naturales) \& PRO-MANU (Proyecto Aprovechamiento y Manejo Sostenible de la Reserva de Biosfera y Parque Nacional del Manu). 2004. Plan maestro del Parque Nacional del Manu. INRENA y PRO-MANU, Cusco. Parte I y II. (acceso en http://sinia. minam.gob.pe/index.php?idElementoInformacion=268)

Silman M.R., A.A. Araujo-Marukami., D.H. Urrego., et al. 2005. Estructura de las comunidades de árboles en el límite sur de la Amazonía occidental: Manú y Madidi. Ecología en Bolivia. Vol. 40(3): 443 - 452.

Ter Steege H., D. Sabatier, H. Castellanos, et al. 2000. An analysis of the floristic composition and diversity of Amazonian forests including those of the Guiana Shield. J. Trop. Ecol. 16: 801-828.

Valencia R., R.B. Foster, G. Villa, R. Condit, et al. 2004. Tree species distributions and local habitat variation in the Amazon: Large forest plot in eastern Ecuador. J. Ecol. 92: 214-229.

Vásquez M.R \& O.L. Phillips. 2000. Allpahuayo: Floristics, structure, and dynamics of a high-diversity forest in Amazonian Peru. Ann. Mo. Bot. Gard. 87: 499-527. 


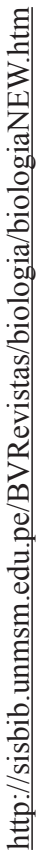

